\title{
High Level of IL-10 in Cerebrospinal Fluid is Specific for Diagnosis of Primary Central Nervous System Lymphoma
}

This article was published in the following Dove Press journal:

Cancer Management and Research

\author{
Jie Shao $\mathbb{D}^{1, *}$ \\ Kun Chen ${ }^{2, *}$ \\ Qing Li (D) \\ Jingjing $M a \mathbb{D}^{\prime}$ \\ Yan $\mathrm{Ma}^{3}$ \\ Zhiguang $\operatorname{Lin}^{3}$ \\ Hui Kang' \\ Bobin Chen ${ }^{3}$ \\ 'Department of Hematology, Huashan \\ Hospital North, Fudan University, \\ Shanghai 201907, People's Republic of \\ China; ${ }^{2}$ Department of Laboratory \\ Medicine, Huashan Hospital North, \\ Fudan University, Shanghai 201907, \\ People's Republic of China; ${ }^{3}$ Department \\ of Hematology, Huashan Hospital, Fudan \\ University, Shanghai 200040, People's \\ Republic of China \\ *These authors contributed equally to \\ this work
}

Purpose: The pathological diagnosis of primary central nervous system lymphoma (PCNSL) by stereotactic brain biopsy and craniotomy is not often applicable due to the high cost and associated complications. In recent years, some biomarkers in cerebrospinal fluid (CSF), including interleukin 10 (IL-10), microRNAs, CXC chemokine ligand 13 (CXCL13), have been reported to be associated with PCNSL. However, this conclusion was controversial. Therefore, this study was to test whether Th17 cell-related cytokines could be used to distinguish PCNSL from other brain tumors.

Patients and Methods: Th17 cell-related cytokines in CSF were measured in 108 patients with intracranial tumors, which included 66 PCNSL patients and 42 patients with other types of brain tumors. A receiver-operating characteristic (ROC) curve was utilized to analyze the diagnostic value of the cytokines based on the area under the curve (AUC).

Results: The CSF IL-10 level and IL-10/IL-6 ratios were significantly higher in PCNSL than in the other brain tumors $(58.2 \mathrm{pg} / \mathrm{mL}$ VS $1.5 \mathrm{pg} / \mathrm{mL}, p=0.001 ; 24.3 \mathrm{VS} 0.6, p=0.001)$. When the cutoff level of IL-10 was set at $8.3 \mathrm{pg} / \mathrm{mL}$, its sensitivity and specificity for diagnosing PCNSL were $59.0 \%$ and $98 \%$, respectively. The CSF IL-10 levels over $5 \mathrm{pg} / \mathrm{mL}$ (+LR 12.3) were of significant value for the diagnosis of PCNSL. These parameters are highly valuable in PCNSL diagnosis, but their sensitivity is less valuable. The sensitivity of IL-4 and IL-17A, the ratio of mature lymphocytes and the monocytes/macrophages ratio in CSF were relatively high. In combination, the sensitivity increased by $15 \%$ and the specificity remained above $85 \%$. The best combination was IL-10 and IL-17A, whose sensitivity was $70 \%$ and specificity was $96 \%$.

Conclusion: The CSF level of IL-10 is a useful diagnostic biomarker in patients with PCNSL. The CSF levels of IL-4, IL-17A, mature lymphocytes and monocytes/macrophages can be used to increase the diagnostic value of CSF IL-10 level and IL-10/IL-6 ratio.

Keywords: primary central nervous system lymphoma, diagnosis, cytokines, IL-10

\section{Introduction}

Primary central nervous system lymphoma (PCNSL) is an aggressive form of nonHodgkin's lymphoma (NHL), and approximately 95\% of PCNSLs are diffuse large B-cell lymphomas (DLBCLs), ${ }^{1}$ which are morphologically indistinguishable from systemic DLBCL. The diagnosis of PCNSL often requires stereotactic brain biopsy and craniotomy, a highly invasive procedure with a $10 \%$ to $35 \%$ rate of diagnostic failure. ${ }^{2}$ The diagnosis rates, mortality and morbidity rates depend on the experience of the surgical team and the neuropathologist. ${ }^{5,7}$ Moreover, many lesions are not amenable to biopsy because of the small size, location in deep brain structures,
Correspondence: Bobin Chen Department of Hematology, Huashan Hospital, Fudan University, Shanghai 200040, People's Republic of China Tel +86-2I-52889999

Email bbchen@fudan.edu.cn 
risk of hemorrhage and other comorbidities. Early and less invasive diagnostic methods for PCNSL represent a challenge.

Th17 cells, a subset of CD4+ T cells, secrete interleukin 17 (IL-17), interleukin 10 (IL-10), interleukin 6(IL-6) and play critical roles in the pathogenesis of inflammatory and autoimmune diseases. The antitumor or protumor roles of Th17 cells in human tumor immunity, however, remain largely unknown and debatable ${ }^{8}$. In addition, Th17 cells and IL-17 were elevated in human myeloma. The frequencies of Th17 cells and the levels of related cytokines (IL-17, IL-6, and TGF- $\beta 1$ ) were increased in patients with acute myeloid leukemia (AML). ${ }^{16,17}$ Th17 cells and the associated cytokines were significantly lower in the peripheral blood from DLBCL patients than those in other cancer patients. In addition, the frequency of Th17 cells in the peripheral blood significantly increased in relapsed patients. ${ }^{14,19}$

Several studies have suggested that cerebrospinal fluid (CSF) molecules, such as microRNAs (miR-21, miR-19, and miR92a), IL-10, and CXC chemokine ligand 13 (CXCL13), may be diagnostic biomarkers for PCNSL. ${ }^{9,10,11,12}$ Vitreoretinal lymphoma (VRL) is a subset PCNSL with a poor prognosis. IL-10 is a cytokine that is expressed by Th17 cells and is a documented biomarker for the diagnosis of $\mathrm{VRL}^{20}$. IL10 levels in the vitreous and aqueous humor (AH) have been shown to be elevated in patients with VRL and to be much higher than those in patients with uveitis. ${ }^{22}$

$\mathrm{IL}-10$ plays a role in lymphoma development, serving as a growth factor for B lymphocytes and as an inducer of the antiapoptotic Bcl-2 pathway. IL-6 is a lymphoid growth- related and differentiation-related cytokine involved in the regulation of immunity and inflammation. Accumulating evidence has indicated that the IL-10 level and the IL-10/IL-6 ratio in CSF were significantly higher in PCNSL patients and may represent a potential diagnostic biomarker for PCNSL ${ }^{23,24}$. However, these results are controversial due to the small number of samples, research design defects and so on. The aim of our study was to investigate the diagnostic value of Th17 cell-related cytokines in CSF for PCNSL.

\section{Patients and Methods Patients and Clinical Data}

A total of 108 patients with suspected PCNSL based on radiographic examinations in our institution from August 2013 through June 2017 were admitted.
Preoperative diagnostic procedures including magnetic resonance imaging (MRI) and/or computed tomography (CT) were completed. The tumor of the patients was confined to the brain, pia mater, spinal cord or eyes, and no other parts of the body were involved. To obtain a pathological diagnosis, brain biopsies, tumor removal, and lumbar puncture were performed after obtaining written informed consent. The final diagnosis of PCNSL was made based on histopathologic analysis of brain $(n=105)$ and cytology analysis of cerebrospinal fluid $(\mathrm{n}=3)$. This study included 66 PCNSLs and 42 other types of brain tumors. Clinical data such as the CSF leukocyte levels, the CSF protein levels, the CSF mature lymphocytes radios, the CSF monocytes/macrophages radios, serum LDH levels, serum $\beta 2-\mathrm{MG}$ levels were collected. Corticosteroid treatment and disease status of PCNSL patients were recorded at the time of each CSF puncture.

\section{Methods}

One milliliter of CSF was drawn from the patients by lumbar puncture after obtaining informed consent and before surgery or biopsies. The CSF samples were centrifuged and frozen within 2 hours of collection, then stored at $-80^{\circ} \mathrm{C}$. The CSF levels of Th17 cell-related cytokines (including IL-1 $\beta$, IL-4, IL-6, IL-10, IL-17A, IL-17F, IL21, IL-22, IL-23, IL-25, IL-31, IL-33, IFN- $\gamma$, sCD40L, TNF- $\alpha$ and so on) were measured in 108 patients by BioPlex Pro ${ }^{\mathrm{TM}}$ Th17 Cytokine Assay produced by Bio-Rad Laboratories, Inc. The assay principle is similar to that of a sandwich ELISA. Data from the reactions were acquired using a Bio-Plex system or a similar Luminex-based reader.

\section{Statistical Analysis}

Differences between the groups were analyzed using the Mann-Whitney $U$-test. $\mathrm{P}<0.05$ was considered statistically significant. A receiver-operating characteristic (ROC) curve was drawn, and the diagnostic value of these cytokines was judged according to the area under the curve (AUC). The Youden index was used to help select the optimum cutoff values, and likelihood ratios were used to assess the IL-10 threshold value in the CSF. Two factors were combined with logistic regression analysis. The ROC curve was used to explore the value of the combined factors. Statistical analysis was performed using SPSS version 22.0 software package. 


\section{Results}

\section{Clinical Characteristics and ThI7}

Cell-Related Cytokine Levels in the CSF of 108 Patients

108 patients suspected PCNSL based on radiographic examinations were admitted. Of the 66 patients with PCNSL, 51 patients were DLBCL, 9 patients were B cell lymphoma, and 6 patients were unclassified PCNSL. Of the 42 patients with other brain tumor types, 2 patients had glioblastoma and 40 patients had metastatic tumors.

The mean age of PCNSL patients was 55 years, whereas the mean age was 52 years for other brain tumor patients. Men represented $61 \%$ of patients in the lymphoma group and $43 \%$ of patients in the other brain tumors group. There were no significant differences in age $(\mathrm{t}=1.20, P=0.24)$ or sex $(\mathrm{t}=1.20, P=0.24)$ between the two groups (Table 1$)$.

The clinical characteristics of 66 PCNSL patients were examined, including 42 males and 24 females. The median age of patients was 55 years old. Most PCNSLs (77.3\%) are diffuse large B-cell lymphomas. Most lesions were located in frontal lobe, followed by parietal lobe, temporal lobe, corpus callosum and lateral ventricle. Patients with serious central infection, intracranial hemorrhage, coagulation disorder, lumbar deformity were not admitted. A small number of patients received low-dose corticosteroids before lumbar puncture to relieve symptoms, detailed data not available. 56 Patients $(84.8 \%)$ received chemotherapy alone, the rest both radiotherapy and chemotherapy, but no radiotherapy alone. The chemotherapy was based on high-dose methotrexate (HD-MTX). Some patients with CSF involvement were treated by intrathecal injection. The complete remission rate after three courses of treatment was $60.3 \%$. The median progression free survival time was 18.7 months (Table 2).

Table I Sex and Age Characteristics of 108 Patients

\begin{tabular}{|c|c|c|c|}
\hline Diagnosis & $\begin{array}{l}\text { Subjects } \\
\text { (n) }\end{array}$ & $\begin{array}{l}\text { Men } \\
(\%)\end{array}$ & $\begin{array}{l}\text { Mean Age } \\
\text { (土SD) }\end{array}$ \\
\hline PCNSL & 66 & 40 & 55 \\
\hline DLBCL & 51 & & \\
\hline B cell lymphoma & 9 & & \\
\hline unclassified PCNSL & 6 & & \\
\hline Other brain & 42 & 18 & 52 \\
\hline tumors & & & \\
\hline glioblastoma & 2 & & \\
\hline metastatic tumors & 40 & & \\
\hline
\end{tabular}

Abbreviations: PCNSL, primary central nervous system lymphoma; DLBCL, diffuse large B-cell lymphoma.
Table 2 Baseline Characteristics of 66 PCNSL Patients

\begin{tabular}{|c|c|}
\hline Baseline Characteristics & $n(\%)$ \\
\hline \multicolumn{2}{|l|}{ Age (years) } \\
\hline$\leq 55$ & $32(48.5 \%)$ \\
\hline$>55$ & 34 (5I.5\%) \\
\hline \multicolumn{2}{|l|}{ Gender } \\
\hline Female & $24(36.4 \%)$ \\
\hline male & $42(63.6 \%)$ \\
\hline \multicolumn{2}{|l|}{ KPS } \\
\hline $0-1$ & $43(65.2 \%)$ \\
\hline$\geq 1$ & $23(34.8 \%)$ \\
\hline \multicolumn{2}{|l|}{ Deep lesion } \\
\hline Yes & $56(84.8 \%)$ \\
\hline No & $10(15.2 \%)$ \\
\hline \multicolumn{2}{|l|}{ CSF Nuclear Cells } \\
\hline Norma I $\left(\leq 8 \times 10^{6} / \mathrm{L}\right)$ & 49 (74.2\%) \\
\hline High $\left(>8 \times 10^{6} / \mathrm{L}\right)$ & $17(25.8 \%)$ \\
\hline \multicolumn{2}{|l|}{ CSF Protein } \\
\hline Normal $(\leq 0.45 \mathrm{~g} / \mathrm{L})$ & $24(36.4 \%)$ \\
\hline High $(>0.45 g / L)$ & 42 (63.6\%) \\
\hline \multicolumn{2}{|l|}{ Eyes Involvement } \\
\hline Yes & 13 (19.7\%) \\
\hline No & 49 (74.2\%) \\
\hline \multicolumn{2}{|l|}{ CSF Lymphoma Cells } \\
\hline Yes & 25 (37.9\%) \\
\hline No & $41(62.1 \%)$ \\
\hline \multicolumn{2}{|l|}{ Spinal lesion } \\
\hline Yes & $5(7.6 \%)$ \\
\hline No & $56(84.8 \%)$ \\
\hline \multicolumn{2}{|l|}{ Treatment } \\
\hline $\mathrm{RT}+\mathrm{CT}$ & $6(9.1 \%)$ \\
\hline $\mathrm{CT}+\mathrm{RT}$ & $4(6.1 \%)$ \\
\hline CT & 56 (84.8\%) \\
\hline \multicolumn{2}{|l|}{ Surgical excision } \\
\hline Yes & $26(39.4 \%)$ \\
\hline No & $40(60.6 \%)$ \\
\hline \multicolumn{2}{|l|}{ Intrathecal injection } \\
\hline Yes & 25 (37.9\%) \\
\hline No & $4(6.1 \%)$ \\
\hline
\end{tabular}

Abbreviations: CT, chemotherapy; RT, radiotherapy.

The CSF IL-10 levels and IL-10/IL-6 ratios were significantly higher in patients with PCNSLs than in patients with other brain tumors $(58.2 \mathrm{pg} / \mathrm{mL}$ VS $1.5 \mathrm{pg} / \mathrm{mL}, p=0.001 ; 24.3$ VS 0.6, $p=0.001$ ). In addition, the CSF mature lymphocyte ratio and the CSF monocyte/macrophage ratio were significantly different between the two groups. The CSF IL-6 
Table 3 Clinical Characteristics and ThI7 Cell-Related Cytokine Levels in the CSF of 108 Patients

\begin{tabular}{|c|c|c|c|c|c|c|}
\hline & $\begin{array}{l}\text { PCNSL (66) } \\
\text { Mean }\end{array}$ & Other Tumors (42) Mean & $P$ value & $\begin{array}{l}\text { PCNSL (66) } \\
\text { Median (P25, P75) }\end{array}$ & $\begin{array}{l}\text { Other Tumors (42) } \\
\text { Median (P25, P75) }\end{array}$ & $P$ value \\
\hline $\mathrm{IL}-\mathrm{I} 0$ (pg/mL) & $58.2 \pm|4| .0$ & $1.5 \pm 1.6$ & 0.001 & $\begin{array}{l}56 \\
(2,89)\end{array}$ & $0.8(0.1,2.5)$ & 0.001 \\
\hline IL-6 (pg/mL) & $12.7 \pm 59.2$ & $5.6 \pm 8.9$ & 0.55 & $13(0.8,16.4)$ & $4.3(0.9,8.0)$ & 0.55 \\
\hline IL-I0/IL-6 & $24.3 \pm 83.2$ & $0.6 \pm 1.1$ & 0.001 & $25(0.7,43.9)$ & $0.2(0.1,0.6)$ & 0.001 \\
\hline IL-4 (pg/mL) & $8.7 \pm 2.3$ & $8.2 \pm 3.0$ & 0.96 & $8.5(7.5,8.8)$ & $7.6(7.4,8.0)$ & 0.96 \\
\hline IL-I7A (pg/mL) & $3.5 \pm 3.5$ & $3.0 \pm 3.9$ & 0.49 & $6.2(1.0,9.4)$ & $1.0(0.9,4.3)$ & 0.49 \\
\hline IL-22 (pg/mL) & $9.1 \pm 6.9$ & $8.6 \pm 6.9$ & 0.89 & $9.5(2.8,15.2)$ & II.3 $(0.9,15.0)$ & 0.89 \\
\hline $\mathrm{sCD} 40 \mathrm{~L}(\mathrm{pg} / \mathrm{mL})$ & $8.1 \pm 7.2$ & $7.9 \pm 7.7$ & 0.94 & $8.6(0.8,14.5)$ & $7.5(0.2,13.9)$ & 0.94 \\
\hline TNF- $\alpha(p g / m L)$ & $1.7 \pm 1.7$ & $1.2 \pm 1.3$ & 0.49 & I.7 $(0.3,2.6)$ & $0.9(0.06,1.9)$ & 0.49 \\
\hline CSF protein $(\mathrm{mg} / \mathrm{l})$ & $0.7 \pm 0.5$ & $0.8 \pm 0.7$ & 0.67 & $0.5(0.4,0.8)$ & $0.4(0.3,0.8)$ & 0.67 \\
\hline CSF WBC $\left(\times 10^{6} / \mathrm{l}\right)$ & $13 \pm 39$ & $13 \pm 28$ & 0.06 & $3(1,8)$ & $5(1,13)$ & 0.06 \\
\hline Serum LDH (U/I) & $190 \pm 57$ & $195 \pm 58$ & 0.56 & $176(155,218)$ & $183(155,217)$ & 0.56 \\
\hline Serum $\beta 2-M G(\mathrm{mg} / \mathrm{l})$ & $1.6 \pm 0.5$ & $1.8 \pm 0.7$ & 0.14 & $1.6(1.3,1.8)$ & $1.6(1.4,2.0)$ & 0.14 \\
\hline CLR (\%) & $80 \pm 21$ & $67 \pm 30$ & 0.04 & $\begin{array}{l}85 \\
(77,93)\end{array}$ & $\begin{array}{l}77 \\
(48,90)\end{array}$ & 0.04 \\
\hline CMR (\%) & $8 \pm 9$ & $16 \pm 16$ & 0.01 & $5(2,10)$ & $12(5,28)$ & 0.01 \\
\hline
\end{tabular}

Abbreviations: CSF, cerebrospinal fluid; CLR, CSF mature lymphocyte ratio; CMR, CSF monocyte/macrophage ratio.

levels were higher in patients with PCNSLs than in patients with other brain tumors, but there was no significant difference (12.7 pg/mL vs $5.6 \mathrm{pg} / \mathrm{mL}, p=0.55$ ) (Table 3).

\section{The AUC of ThI7 Cell-Related Cytokine CSF Levels in 108 Patients}

The ROC curve was generated and the diagnostic value of these cytokines was evaluated according to the AUC. The Youden index was used to help select the optimum cutoff values. The AUC of the CSF IL-10 levels and the IL-10/IL-6 ratio was over 0.8 (Figures 1 and 2), which represented a significant diagnostic value for PCNSL. At an IL-10 cutoff

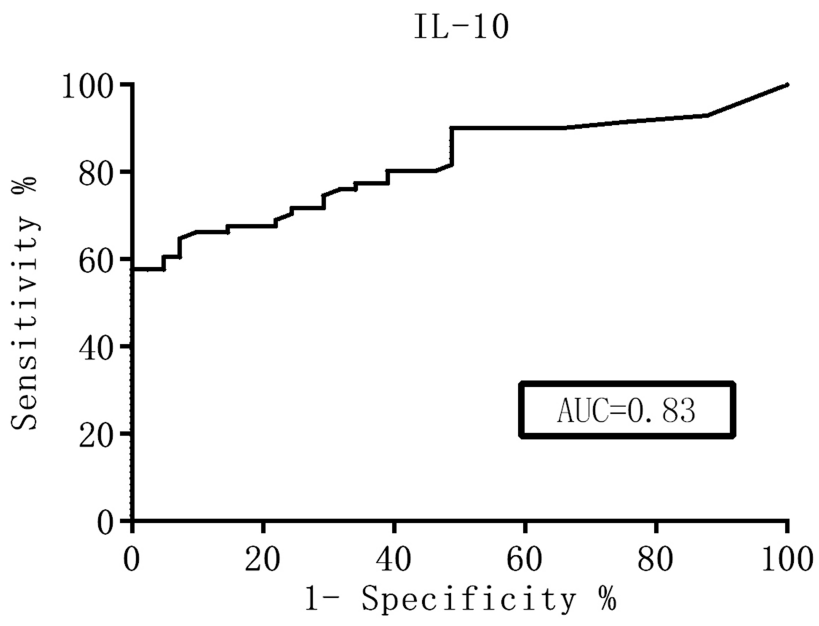

Figure I ROC curves for the IL-I0 CSF levels. level of $8.3 \mathrm{pg} / \mathrm{mL}$, the sensitivity and specificity were $59.0 \%$ and $98 \%$, respectively. At an IL-10/IL-6 cutoff of 1.6, the sensitivity and specificity were $66.0 \%$ and $91 \%$, respectively. These parameters were highly valuable for PCNSL diagnosis, but their sensitivity was not satisfactory. The AUC of the CSF IL-6, L-22, sCD40L, and TNF- $\alpha$ levels and the other factors were near 0.5 , with no significant diagnostic value (Table 4).

\section{Statistical Indices for the IL-I0, IL-I0/IL-6,} IL-I7A Threshold Values in CSF

Likelihood ratios were used to assess the threshold value of IL-10 CSF levels. The positive likelihood ratio of cytokines over 10 indicated a significant diagnostic value. When IL-10 CSF levels exceeded $5 \mathrm{pg} / \mathrm{mL}$, the positive likelihood ratio was 12.3 , which was a significant diagnostic value. The positive likelihood ratios were 6.0 and 3.4 when the CSF IL-10/IL-6 ratio was greater than 1 and when IL-17A CSF levels were between 5-10 pg/mL, respectively, which were not statistically significant (Table 5-7).

\section{The Area Under the ROC Curve of the Combined Factors}

Data before showed that the CSF IL-10 level and IL-10/IL-6 ratio were significantly higher in PCNSL but less sensitive. The sensitivity of IL-4 and IL-17A, the ratio of mature lymphocytes and the monocytes/macrophages ratio in CSF were relatively high. Therefore, two factors were combined to generate a new diagnostic factor with logistic regression 


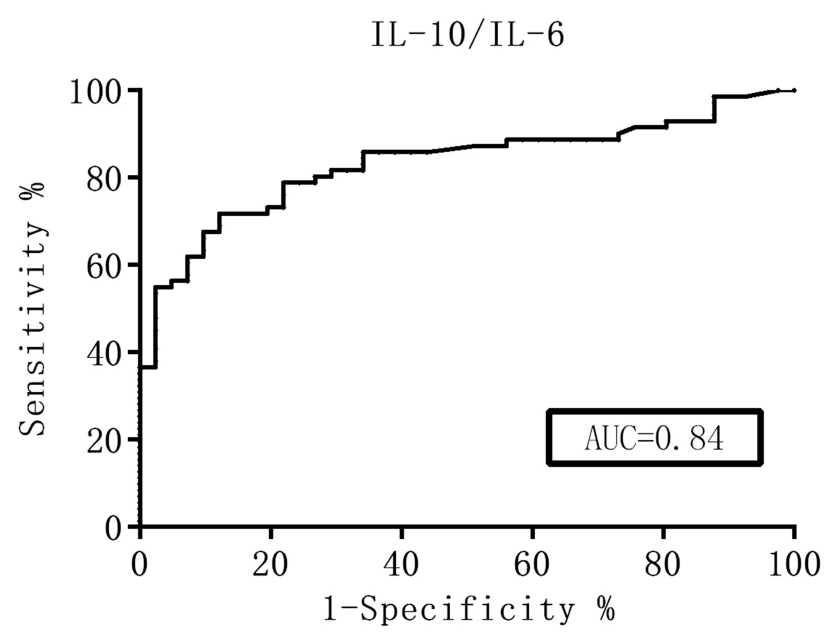

Figure 2 ROC curves for the CSF IL-10/IL-6 ratio.

analysis. The ROC curve was used to explore the value of the combined factors. In combination, the sensitivity increased by $15 \%$, and the specificity remained above $85 \%$. The best combination was IL-10 and IL-17A, whose sensitivity was $70 \%$ and specificity was $96 \%$ (Table 8 ).

In summary, high level of IL-10 and high radio of IL10/IL-6 in cerebrospinal fluid was specific for diagnosis of primary central nervous system lymphoma. The CSF IL-4 levels, the CSF IL-17A levels, the CSF mature lymphocytes ratios, the CSF monocytes/macrophages ratios were of high sensitivity. In combination, the sensitivity reached $70 \%$, and the specificity remained above $85 \%$. The best

Table 4 The AUC of ThI7 Cell-Related Cytokine CSF Levels in 108 Patients

\begin{tabular}{|l|l|l|l|l|}
\hline & AUC & Cutoff & Se (\%) & Sp (\%) \\
\hline IL-I0 (pg/mL) & 0.83 & 8.3 & 59 & 98 \\
IL-6 (pg/mL) & 0.50 & 4.2 & 30 & 67 \\
IL-I0/IL-6 & 0.84 & 1.6 & 66 & 91 \\
IL-4 (pg/mL) & 0.62 & 8.8 & 30 & 79 \\
IL-I7A (pg/mL) & 0.58 & 4.7 & 67 & 81 \\
IL-22 (pg/mL) & 0.57 & 13.6 & 53 & 52 \\
SCD40L (pg/mL) & 0.55 & 13.3 & 46 & 55 \\
TNF- $\alpha(\mathrm{pg} / \mathrm{mL})$ & 0.58 & 1.9 & 37 & 79 \\
CSF protein (mg/l) & 0.55 & 0.63 & 31 & 63 \\
CSF WBC (xI0 $/ \mathrm{l})$ & 0.43 & 24 & 12 & 87 \\
Serum LDH (U/l) & 0.50 & 185 & 45 & 55 \\
Serum $\beta 2-M G(\mathrm{mg} / \mathrm{l})$ & 0.41 & 1.65 & 39 & 50 \\
CLR $(\%)$ & 0.63 & 78 & 73 & 50 \\
CMR (\%) & 0.72 & 4.5 & 48 & 76 \\
\hline
\end{tabular}

Abbreviations: CLR, CSF mature lymphocyte ratio; CMR, CSF monocyte/macrophage ratio; AUC, area under the curve; Se, sensitivity; Sp, specificity.
Table 5 Statistical Indices for the IL-10 Threshold Values in CSF

\begin{tabular}{|l|l|l|l|}
\hline \multirow{2}{*}{ IL-I O (pg/mL) } & \multicolumn{2}{|l|}{ Total } \\
\cline { 2 - 4 } & PCNSL (\%) & Other Brain Tumors (\%) & +LR \\
\hline$<1$ & $13(19)$ & $21(50)$ & 0.37 \\
$1-3$ & $8(I I)$ & $12(28)$ & 0.40 \\
$3-5$ & $8(I I)$ & $7(17)$ & 0.69 \\
$\geq 5$ & $4 I(59)$ & $2(5)$ & 12.3 \\
\hline
\end{tabular}

Abbreviation: $+\mathrm{LR}$, positive likelihood ratio.

Table 6 Statistical Indices for the IL- 10/IL-6 Threshold Values in the CSF

\begin{tabular}{|l|l|l|l|}
\hline \multirow{2}{*}{ IL-1 0/IL-6 } & \multicolumn{3}{|l|}{ Total } \\
\cline { 2 - 4 } & PCNSL (\%) & Other Brain Tumors (\%) & +LR \\
\hline$<0.1$ & $8(I I)$ & $13(3 \mathrm{I})$ & 0.37 \\
$0.1-0.5$ & $5(7)$ & $17(40)$ & 0.18 \\
$0.5-1$ & $7(10)$ & $7(17)$ & 0.60 \\
$\geq I$ & $50(72)$ & $5(12)$ & 6.00 \\
\hline
\end{tabular}

Table 7 Statistical Indices for the IL- I7A Threshold Values in CSF

\begin{tabular}{|l|l|l|l|}
\hline \multirow{2}{*}{$\begin{array}{l}\text { IL-I7A } \\
\text { (pg/mL) }\end{array}$} & \multicolumn{3}{|l|}{ Total } \\
\cline { 2 - 4 } & PCNSL (\%) & Other Brain Tumors (\%) & +LR \\
\hline$<1$ & $16(23)$ & $14(33)$ & 0.69 \\
$1-5$ & $31(44)$ & $21(50)$ & 0.89 \\
$5-10$ & $17(24)$ & $3(7)$ & 3.40 \\
$\geq 10$ & $6(9)$ & $4(10)$ & 0.90 \\
\hline
\end{tabular}

Table 8 The Area Under the ROC Curve of the Combined Factors

\begin{tabular}{|l|l|l|l|}
\hline Combined Factors & AUC & Se (\%) & Sp (\%) \\
\hline IL-I0+IL-I0/IL-6 & 0.86 & 70 & 92 \\
IL-I0+IL-I7A & 0.90 & 70 & 96 \\
IL-I0+IL-4 & 0.83 & 70 & 85 \\
IL-I0+CLR & 0.85 & 64 & 89 \\
IL-I0/IL-6+IL-I7A & 0.83 & $7 I$ & 89 \\
IL-I0/IL-6+IL-4 & 0.83 & 70 & 85 \\
IL-I0/IL-6+CLR & 0.85 & 68 & 92 \\
\hline
\end{tabular}

Abbreviations: ROC curve, receiver-operating characteristic curve; AUC, area under the curve.

combination was IL-10 and IL-17A, whose sensitivity was $70 \%$ and specificity was $96 \%$.

\section{Discussion}

PCNSL is a rare NHL whose clinical characteristics are not specific and diagnosis is difficult. More than $90 \%$ PCNSLs are diffuse large B-cell lymphomas, which have 
a poor prognosis. ${ }^{1}$ There is currently no standard treatment for PCNSL. Chemotherapy based on high-dose methotrexate is currently recommended as the first-line treatment. ${ }^{2,4}$ Therefore, early diagnosis is very important for treatment and prognosis of PCNSL.

Currently, the gold standard for PCNSL diagnosis is stereotactic brain biopsy, ${ }^{3}$ which is difficult, expensive and traumatic, with a failure rate of $10 \%-35 \%{ }^{7}$ At the same time, serious complications such as hemorrhage and intracranial infection may occur. ${ }^{6}$ Some patients cannot undergo biopsy because of the small size of the lesion, the deep location, the poor physical condition and so on. In recent years, accumulating studies on PCNSL have been performed. However, the results have been controversial due to the small number of samples, research design defects. The aim of our study was to investigate the diagnostic value of Th17 cell-related cytokines in CSF for primary central nervous system lymphomas.

In recent years, related studies have shown that Th17 cells, as tumor-infiltrating lymphocytes that secrete cytokines such as IL-17, IL-10 and IL-6, exist in the tumor microenvironment of various solid tumors such as nasopharyngeal carcinoma, gastric cancer, colon cancer, pancreatic cancer and ovarian cancer. ${ }^{13,15,16}$ In addition, several studies have shown that Th17 cells are closely related to the development of AML, multiple myeloma and NHL. ${ }^{25,26}$ The frequencies of Th17 cells and the levels of related cytokines (IL-17, IL-6, and TGF- $\beta 1$ ) were increased in patients with AML. Th17 cells and the associated cytokines were significantly lower in the peripheral blood of DLBCL patients than those in other cancer patients. IL-10 is a cytokine expressed by Th17 cells, which were significantly increased in patients with vascular immunoblastic T-cell lymphoma (AITL) $(\mathrm{P}<0.05){ }^{21}$ The clinical symptoms of extranodal NK/T-cell lymphoma (ENKTL) patients were more severe in patients with high serum IL-10 levels $(>12.28 \mathrm{pg} / \mathrm{mL}){ }^{27}$

IL-10 is an immunoregulatory factor that plays an important role in many diseases, such as autoimmune diseases, inflammation, and tumors. IL-10 can be secreted by a variety of immune cells such as monocytes, macrophages, Th2 cells, Th17 cells, B cells, dendritic cells and by non-immune cells. ${ }^{9}$ IL-10, as a growth factor of B lymphocytes, plays an important role in the development of lymphoma by inducing and activating the Bcl-2 antiapoptotic pathway.

In 2012, a retrospective study from Sasayama found that the CSF level of IL-10 was significantly higher in
PCNSL than in other intracranial tumors; the CSF level of IL-10 decreased in all patients after treatment and the CSF level of IL-10 increased again in most recurrent patients. ${ }^{22}$ In 2016, Nguyen-Them and other researchers examined the CSF levels of IL-10 and IL-6 in 79 PCNSL patients and in 40 patients with other central nervous system diseases. The results showed that the CSF levels of IL-6 and the IL-10/IL-6 ratio were not significantly different. ${ }^{29}$ Therefore, the results regarding the diagnostic value of the IL-6 CSF levels and the IL-10/IL-6 ratio are controversial.

In this study, the CSF IL-10 levels and the IL-10/IL-6 ratio were significantly higher in PCNSLs than in the other brain tumors $(58.2 \mathrm{pg} / \mathrm{mL} \mathrm{VS} 1.5 \mathrm{pg} / \mathrm{mL}, p=0.001 ; 24.3 \mathrm{VS}$ $0.6, p=0.001)$. The CSF IL-6 levels were higher in PCNSLs than in other brain tumors, but there was no significant difference $(12.7 \mathrm{pg} / \mathrm{mL}$ vs $5.6 \mathrm{pg} / \mathrm{mL}$, $p=0.55)$. At an IL-10 cutoff level of $8.3 \mathrm{pg} / \mathrm{mL}$, the sensitivity and specificity were $59.0 \%$ and $98 \%$, respectively. At an IL-10/IL-6 cutoff of 1.6, the sensitivity and specificity were $66.0 \%$ and $91 \%$, respectively. The CSF IL-10 levels over $5 \mathrm{pg} / \mathrm{mL}$ (+LR 12.3) was of significant value for the diagnosis of PCNSL. These parameters were highly valuable in PCNSL diagnosis, but their sensitivity was not satisfactory. The reasons for the low sensitivity may be that the sample numbers were not large enough, that different detection methods were used in different studies and that different cutoff points were chosen. When the cutoff point of the CSF IL-10 levels was $2.8 \mathrm{pg} / \mathrm{mL}$, the sensitivity and specificity are $70 \%$ and $76 \%$, respectively.

The sensitivities of the CSF IL-4 and IL-17A levels and the CSF mature lymphocyte and monocyte/macrophage ratios were relatively high. In combination, the sensitivity increased by $15 \%$ and the specificity remained above $85 \%$. The best combination was IL-10 and IL-17A, whose sensitivity was $70 \%$ and specificity was $96 \%$. There have been no relevant studies on the diagnostic value of IL-4 and IL-17A CSF levels. However, studies on NHL have shown that in the peripheral blood of B-cell NHL patients, the Th17 cell level was significantly lower than that in the peripheral blood of healthy people. The plasma level of IL-17AF was significantly reduced, and the IL17AA level did not significantly change. Th17 cell levels in relapsed patients were significantly higher than those in patients before treatment or in healthy people. Researchers believe that changes in Th17 cell levels may be associated with treatment responses, pathological types and tumor stages in B-cell NHL patients. ${ }^{14}$ 
IL-17A is a major inflammatory cytokine secreted by Th17 cells. IL-17A plays an important role in microbial infections. IL-17A also participates in inflammation, autoimmune diseases, metabolic diseases, tumorigenesis and development. In vivo and in vitro experiments performed abroad on IL-17A have shown that IL-17A can further promote the growth of DLBCL tumors by promoting the proliferation and angiogenesis of malignant tumors. ${ }^{16,28}$ However, domestic studies also showed that the Th17 cell level and the IL-17 and IL-21 levels in DLBCL patients were negatively correlated with the International Prognostic Index (IPI) scores. There is no consensus about whether Th17 cells can promote or inhibit the development of tumors. Most evidences show that Th17 cells exert antitumor immune effects in DLBCL. ${ }^{18}$ However, the mechanism is still unclear. Therefore, a large number of in vitro and in vivo experiments are needed to further confirm this conclusion.

\section{Conclusion}

In conclusion, the IL-10 CSF level is a useful diagnostic biomarker for PCNSL patients, less invasive than current diagnostic methods. IL-4, IL-17A, mature lymphocytes, and monocytes/macrophages can contribute to the diagnostic value of IL-10 and the IL-10/IL-6 ratio in CSF. Few studies about the correlation between the IL-4, IL-17A CSF levels and PCNSL have been reported, and the mechanism needs further confirmation.

\section{Ethics}

This research was approved by the Ethics Committee of Huashan Hospital North, Fudan University. All procedures. Followed were in accordance with the ethical standards of the responsible committee on human experimentation (institutional and national) and with the Helsinki Declaration of 1975, as revised in 2008. All the patients voluntarily participated in this study and provided written informed consent. All the participants' personal information is confidential.

\section{Funding}

This study was funded by Shanghai Shenkang Hospital Development Center: Three-year Plan for Promoting Clinical Skills and Innovations in Municipal Hospitals (Project No.16CR2043B), also funded by Beijing Medical And Health Foundation (Project No.YWJKJJHKB175B).

\section{Disclosure}

The authors report no conflicts of interest in this work.

\section{References}

1. Citterio G, Reni M, Gatta G, et al. Primary central nervous system lymphoma. Crit Rev Oncol Hematol. 2017;113:97-110. doi:10.1016/ j.critrevonc.2017.03.019

2. Hoang-xuan K, Bessell E, Bromberg J, et al. Diagnosis and treatment of primary CNS lymphoma in immunocompetent patients: guidelines from the European Association for Neuro-Oncology. Lancet Oncol. 2015;16(7):e322-32. doi:10.1016/S1470-2045(15)00076-5

3. Korfel A, Schlegel U. Diagnosis and treatment of primary CNS lymphoma. Nat Rev Neurol. 2013;9(6):317-327. doi:10.1038/ nrneurol.2013.83

4. Shiels MS, Pfeiffer RM, Besson C, et al. Trends in primary central nervous system lymphoma incidence and survival in the U.S. $\mathrm{Br}$ J Haematol. 2016;174(3):417-424. doi:10.1111/bjh.14073

5. Can SM, Turkmenoglu ON, Tanik C, et al. Computerized tomography-guided stereotactic biopsy of intracranial lesions: report of 500 consecutive cases. Turk Neurosurg. 2017;27(3):395-400. doi:10.5137/1019-5149.JTN.16280-15.1

6. Malone H, Yang J, Hershman DL, et al. Complications following stereotactic needle biopsy of intracranial tumors. World Neurosurg. 2015;84(4):1084-1089. doi:10.1016/j.wneu.2015.05.025

7. Nishihara M, Sasayama T, Kudo H, et al. Morbidity of stereotactic biopsy for intracranial lesions. Kobe J Med Sci. 2011;56(4):E148-53.

8. Kryczek I, Banerjee M, Cheng P, et al. Phenotype, distribution, generation, and functional and clinical relevance of Th17 cells in the human tumor environments. Blood. 2009;114(6):1141-1149. doi:10.1182/blood-2009-03-208249

9. Mabray MC, Barajas RF, Villanueva-meyer JE, et al. The combined performance of $\mathrm{ADC}, \mathrm{CSF} \mathrm{CXC}$ chemokine ligand 13, and csf interleukin 10 in the diagnosis of central nervous system lymphoma. AJNR Am J Neuroradiol. 2016;37(1):74-79. doi:10.3174/ajnr.A4450

10. Rubenstein JL, Wong VS, Kadoch C, et al. CXCL13 plus interleukin 10 is highly specific for the diagnosis of CNS lymphoma. Blood. 2013;121(23):4740-4748. doi:10.1182/blood-2013-01-476333

11. Mao X, Sun Y, Tang J. Serum miR-21 is a diagnostic and prognostic marker of primary central nervous system lymphoma. Neurol Sci. 2014;35(2):233-238. doi:10.1007/s10072-013-1491-9

12. Roth P, Keller A, Hoheisel JD, et al. Differentially regulated miRNAs as prognostic biomarkers in the blood of primary CNS lymphoma patients. Eur J Cancer. 2015;51(3):382-390. doi:10.1016/j.ejca.2014.10.028

13. Liu T, Peng L, Yu P, et al. Increased circulating Th22 and Th17 cells are associated with tumor progression and patient survival in human gastric cancer. J Clin Immunol. 2012;32(6):1332-1339. doi:10.1007/ s10875-012-9718-8

14. Lu T, Yu S, Liu Y, et al. Aberrant circulating Th17 cells in patients with B-cell Non-Hodgkin's Lymphoma. PLoS One. 2016;11(1): e0148044. doi:10.1371/journal.pone.0148044

15. Maruyama T, Kono K, Mizukami Y, et al. Distribution of Th17 cells and FoxP3(+) regulatory $\mathrm{T}$ cells in tumor-infiltrating lymphocytes, tumor-draining lymph nodes and peripheral blood lymphocytes in patients with gastric cancer. Cancer Sci. 2010;101(9):1947-1954. doi:10.1111/j.1349-7006.2010.01624.x

16. Qi W, Huang X, Wang J. Correlation between Th17 cells and tumor microenvironment. Cell Immunol. 2013;285(1-2):18-22. doi:10.101 6/j.cellimm.2013.06.001

17. Ye J, Livergood RS, Peng G. The role and regulation of human Th17 cells in tumor immunity. Am $J$ Pathol. 2013;182(1):10-20. doi:10.1016/j.ajpath.2012.08.041

18. Young MR. Th17 cells in protection from tumor or promotion of tumor progression. J Clin Cell Immunol. 2016;7(3):431. doi:10.4172/ 2155-9899.1000431 
19. Zhong W, Xu X, Zhu Z, et al. Increased expression of IRF8 in tumor cells inhibits the generation of Th17 cells and predicts unfavorable survival of diffuse large B cell lymphoma patients. Oncotarget. 2017;8(30):49757-49772. doi:10.18632/oncotarget.17693

20. Pochat-cotilloux C, Bienvenu J, Nguyen AM, et al. Use of a threshold of interleukin-10 and Il-10/Il-6 ratio in ocular samples for the screening of vitreoretinal lymphoma. Retina. 2018;38 (4):773-781. doi:10.1097/IAE.0000000000001922

21. Ham JS, Park HY, Ryu KJ, et al. Elevated serum interleukin-10 level and M2 macrophage infiltration are associated with poor survival in angioimmunoblastic T-cell lymphoma. Oncotarget. 2017;8(44):76 231-76240. doi:10.18632/oncotarget.19301

22. Sasayama T, Nakamizo S, Nishihara M, et al. Cerebrospinal fluid interleukin-10 is a potentially useful biomarker in immunocompetent primary central nervous system lymphoma (PCNSL). NeuroOncology. 2012;14(3):368-380. doi:10.1093/neuonc/nor203

23. Wang L, Luo L, Gao Z, et al. The diagnostic and prognostic value of interleukin-10 in cerebrospinal fluid for central nervous system lymphoma: a meta-analysis. Leuk Lymphoma. 2017;58(10):2452-2459. doi:10.1080/10428194.2017.1289523

24. Song Y, Zhang W, Zhang L, et al. Cerebrospinal fluid IL-10 and IL-10/IL-6 as accurate diagnostic biomarkers for primary central nervous system large B-cell lymphoma. Sci Rep. 2016;6:38671. doi: $10.1038 /$ srep38671
25. Gelebart P, Zak Z, Dien-bard J, et al. Interleukin 22 signaling promotes cell growth in mantle cell lymphoma. Transl Oncol. 2011;4 (1):9-19. doi:10.1593/tlo.10172

26. Liao F, Hsu Y-C, Kuo S-H, et al. Genetic polymorphisms and tissue expression of interleukin-22 associated with risk and therapeutic response of gastric mucosa-associated lymphoid tissue lymphoma. Blood Cancer J. 2014;4:eXX. doi:10.1038/bcj.2014.70

27. Wang $\mathrm{H}$, Wang $\mathrm{L}$, Wuxiao $\mathrm{Z}$, et al. Increased serum levels of interleukin-10 predict poor prognosis in extranodal natural killer/ T-cell lymphoma patients receiving asparaginase-based chemotherapy. Onco Targets Ther. 2015;8:2589-2599. doi:10.2147/ OTT.S91077

28. Miyagaki T, Sugaya M, Suga H, et al. IL-22, but not IL-17, dominant environment in cutaneous T-cell lymphoma. Clin Cancer Res. 2011;17(24):7529-7538. doi:10.1158/1078-0432.CCR-11-1192

29. Nguyen-them L, Costopoulos M, Tanguy ML, et al. The CSF IL-10 concentration is an effective diagnostic marker in immunocompetent primary CNS lymphoma and a potential prognostic biomarker in treatment-responsive patients. Eur J Cancer. 2016;61:69-76. doi:10.1016/j.ejca.2016.03.080

\section{Publish your work in this journal}

Cancer Management and Research is an international, peer-reviewed open access journal focusing on cancer research and the optimal use of preventative and integrated treatment interventions to achieve improved outcomes, enhanced survival and quality of life for the cancer patient.
The manuscript management system is completely online and includes a very quick and fair peer-review system, which is all easy to use. Visit http://www.dovepress.com/testimonials.php to read real quotes from published authors. 